\title{
Gold Sensitization in Photography
}

\author{
ADVANCES IN KNOWLEDGE OF ITS MECHANISM
}

\author{
Wolfgang $\mathbf{F}$. Berg \\ Department of Photography, Swiss Federal Institute of Technology (ETHZ), Zurich, Switzerland
}

An earlier article in Gold Bulletin (1) has reviewed the various uses of gold in the photographic process. Since it was written, some progress has been made in understanding the mechanism of the most important application of gold in photography, namely its use to increase the sensitivity of silver halide crystals to light. The concentrations of gold necessary are minute, of the order of ppm. If one estimates the total amount of silver used in photographic materials as a few thousand tons/year, then in the same period around $1 \mathrm{~kg}$ of gold is used, which is so little that the loss in making up solutions etc., is likely to exceed vastly the amount which is incorporated in photographic films.

\section{The Latent Image}

The very smallness of the amount of gold involved in photographic emulsions at once points to the part it must play. To appreciate this, one must briefly consider the elementary process of formation of the latent image in which a very weak exposure profoundly increases the rate of reduction of the light-sensitive crystals of a film or paper in the developer and so transforms the latent into a visible image.

The emulsion crystals each contain between $10^{8}$ and $10^{9}$ ions of silver together with their associated halide (bromide and/or chloride and/or iodide) ions. In latent image formation, exposure changes a very few $\mathrm{Ag}^{+}$ions to silver atoms (and a corresponding number of halide $\mathrm{X}^{-}$ions to halogen atoms), by a process described as photolysis. It was early recognized that for this small number of silver atoms to be effective, they must assemble or coagulate to form small specks: the latent image or development centres. These may contain as few as four silver atoms $(2,3)$.

\section{Chemical Sensitization}

Ellis (1) has described how the sensitivity of the emulsion crystals is increased by the technological step of chemical sensitization: a heat treatment in the presence of certain chemicals, in which the size of the crystals remains virtually unchanged, but whereby the formation of the latent image is made more effective. There are only three ways imaginable by which such a sensitization could occur (4):

(1) Photolytic sensitization (PS): where the possible recombination of silver and halogen atoms to re-form silver halide $\mathrm{Ag}^{+} \mathrm{X}^{-}$is impeded. In the reversible process

$$
\mathrm{Ag}^{+} \mathrm{X}^{-}+\text {light } \underset{\text { recombination }}{\stackrel{\text { photolysis }}{\longrightarrow}} \mathrm{Ag}+\mathrm{X}
$$

the reverse reaction is inhibited by PS.

(2) Topological sensitization (TS): in which the latent image centre is made to form where it is wanted. As a rule it is needed on the crystal surface, where the normal developer can contact it. With the $\mathrm{Ag}^{+} \mathrm{Br}^{-} / \mathrm{I}^{-}$crystals of a high-speed film, latent image centres will tend to form in the grain interior unless TS is carried out. The most common - and indeed most important - example of TS is that by sulphur compounds, which is usually effected by heat treatment in the presence of ppm amounts of thiosulphate, $\mathrm{S}_{2} \mathrm{O}_{3}^{2-}$. In photographic jargon this heat treatment is known as 'digestion', and it raises sensitivity by a factor of 10 or more. With certain emulsions, in which the majority of unsensitized crystals would not respond to light at all, sulphur sensitization ensures that they all take part in forming surface latent image.

(3) Development sensitization (DS): whereby chemicals cause a silver centre too small to induce development to become effective. A centre which is too small is known as a sub-image centre, and its transformation into a development centre as latensification. DS can often be carried out after exposure, and where the sensitizing chemicals are present already before exposure, they may well act only after the latent image silver is formed. Gold sensitization would appear to be a typical case of DS, but the complete story is more involved.

\section{The Fate of Gold in the Emulsion}

In view of the minute quantity in which gold is added to photographic emulsions it is not surprising that little is known about its chemical role. The amount of gold present in an emulsion is so small that it can be detected conveniently only by optical spectroscopic analysis. To study the involvement of it in the process, activation analysis can be used; another useful method is based on the use of the radio-active 
isotope gold-198. This was utilized in recent work by Faelens (5) who attempted to check whether, and in what form, the gold was held by the emulsion crystals and to what extent it was present in the surrounding gelatine matrix. He showed that on digestion with gold (I) thiocyanate complex $\mathrm{Au}(\mathrm{CNS})_{2}^{-}$, both in the presence or absence of small amounts of the reducing agent thiourea dioxide, no measurable amount of gold stays on the grains. Moreover, on digestion with additional thiosulphate $\mathrm{S}_{2} \mathrm{O}_{3}^{2-}$, which on its own would form silver sulphide $\mathrm{Ag}_{2} \mathrm{~S}$, increasing amounts of gold are fixed by the grains with increasing time of digestion until a steady condition is reached. Faelens suggests that with thiocyanate-digestion, the gold must act in the form of atoms so small in number that they defy detection, whereas with thiosulphate-digestion, gold sulphide or perhaps a mixed silver-gold sulphide may be formed. But here also, the effective gold may be in the form of atoms. It may be that the effective gold is, in fact, present in the gelatin and that digestion, while technologically essential, has but minor effects on that part of sensitization due to the gold.

This work must be regarded as a valuable useful start, but further microchemical work coupled with detailed photographic testing will be required before the chemical condition of the gold in photographic systems is seen more clearly. This is a pre-condition for understanding its function and, at present, any theorizing has to be regarded as speculative.

\section{Stability of the Latent Image}

That a speck consisting of as few as four silver atoms should be stable for any length of time is unexpected; one would anticipate that it would be readily oxidized. In general, small particles of any material are less stable than large ones, their excess energy, which is a measure of instability, being determined by their surface to volume ratio, in accord with the Gibbs-Thomson law. Stability also depends upon the environment. Thus, if latent image is formed in an aqueous suspension or hydrosol of silver halide, it is lost rapidly, with a half life of around 10 seconds $(6,7,8)$. The addition of gelatin at once ensures stability, the amount necessary being of the order of that required to cover monomolecularly the surfaces of the crystals. In electrochemical terms, gelatin makes the silver centres more noble, that is less ready to give up electrons to an oxidizing agent. Recent work (8) indicates that very small fractions of gelatin molecules can effect this stabilization.

It would be surprising if the Gibbs-Thomson law were to apply strictly to particles consisting of just a few atoms (9). Indeed, calculations $(10,11)$ and experiments (12) indicate that an $\mathrm{Ag}_{2}$ unit may be more stable than an $\mathrm{Ag}_{3}$ unit. Even beyond $\mathrm{Ag}_{4}$, stability is supposed to increase in an irregular manner.
Since $\mathrm{Au}^{+}$ions in contact with silver may be reduced to gold atoms, a silver centre is likely to turn into one of gold and thus become more noble, in which form it is more resistant to oxidation and more ready to accept electrons from a developer. The gold latent image is a better development centre.

Experiments on silver bromide hydrosols (4) have demonstrated that a latent image will fade more rapidly than a sub-image (presumably associated with $\mathrm{Ag}_{2}$ centres) and that, as is well known with emulsions $(13,14)$, a sub-image can be turned into development centres through latensification by adding $\mathrm{Au}^{+}$after exposure. The work has further shown that if $\mathrm{Au}^{+}$is present before exposure, sensitivity is increased more effectively than by addition of $\mathrm{Au}^{+}$ afterwards.

To summarize, one can formulate a mechanism of gold sensitization in which gold exercises two distinct functions which are closely related through processes of electrochemical stabilization (15). Firstly, sensitization during exposure comes about because the small growing silver centre is stabilized at a stage when it might otherwise be subject to regression. Thus, latent image is built up more effectively: a case of photolytic sensitization. Secondly, a silver centre too small to be able to induce development (sub-image) may become a development centre if converted to gold. This is especially valuable when very brief times of exposure are involved, such as with electronic flash, laser-beam writing, or scanners in graphic-arts work, since under these conditions a large proportion of the silver centres formed would be very small and thus constitute a non-developable sub-image. Gold converts this to developable latent image. The resulting increase in sensitivity is from two to three times and is a most valuable effect additional to chemical sensitization by sulphur compounds and has made possible the introduction of available-light photography both in black-and-white and in colour.

\section{References}

1 P. Ellis, Gold Bull., 1975, 8, (1), 7.12

2 G. C. Farnell and J. B. Chanter, f. Photogr. Sci., 1961, 9, 73

3 A. Marriage, f. Photogr. Sci., 1961, 9, 93

4 O. Tanabe and W. F. Berg, f. Photogr. Sci., 1977, 25, 45

5 P. Faelens, 7. Photogr. Sci., 1978, 26, 144

6 H. Keller, Photogr. Korr., 1967, 103, 69, 86, 104, 117

7 W. F. Berg and H. E. Keller, Photogr. Sci. Eng., 1967, 11, 178

8 T. Y. Wang, I. Tomka and W. F. Berg, to be published

9 see, however, I. Konstantinow and J. Malinowski, f. Photogr. Sci., 1975, 23, 145

10 R. C. Baetzold, Photogr. Sci. Eng., 1975, 19, 11

11 J. W. Mitchell, Photogr. Sci. Eng., 1978, 22, 1 and 249

12 E. Moisar, F. Granzer, D. Dautrich and E. Palm, F. Photogr. Sci., 1977, 25, 12

13 T. H. James et al., PSAf, 1948, 14, 349

14 T. H. James, 7 . Colloid Sci., 1948, 3, 447

15 J. M. Harbison and J. F. Hamilton, Photogr. Sci. Eng., 1975, 19, 332 